\title{
The Weak Galerkin Finite Element Method for Solving the Time-Dependent Integro-Differential Equations
}

\author{
Xiuli Wang ${ }^{1,2}$, Qilong Zhai ${ }^{3}$, Ran Zhang ${ }^{2, *}$ and Shangyou Zhang ${ }^{4}$ \\ ${ }^{1}$ College of Computer Science and Technology, Jilin University, Changchun 130012, \\ Jilin, China \\ 2 School of Mathematics, Jilin University, Changchun 130012, Jilin, China \\ 3 School of Mathematics Science, Peking University, Beijing 100871, China \\ ${ }^{4}$ Department of Mathematics Science, University of Delaware, Newark 19716, USA
}

Received 1 April 2019; Accepted (in revised version) 8 October 2019

\begin{abstract}
In this paper, we solve linear parabolic integral differential equations using the weak Galerkin finite element method (WG) by adding a stabilizer. The semidiscrete and fully-discrete weak Galerkin finite element schemes are constructed. Optimal convergent orders of the solution of the WG in $L^{2}$ and $H^{1}$ norm are derived. Several computational results confirm the correctness and efficiency of the method.
\end{abstract}

AMS subject classifications: 65M60, 65M15, 65M12

Key words: Integro-differential problem, weak Galerkin finite element method, discrete weak gradient, discrete weak divergence.

\section{Introduction}

Integro-differential equations [24] are used to simulate many phenomena in the fields of mathematics, dynamics and engineering technology $[1,9,15]$. It is also used in highenergy physics and biomedicine to help describe related physical phenomena and laws [7, 13]. Especially, in geology, the integro-differential equation [31] can be used to describe the fine three-dimensional problem of the interior of the earth to explore mineral products and predict earthquakes [8]. It also plays an important role in aerodynamics [3]. For example, it blue can be used to study the Brown displacement and thermal diffusion of suspended grain in heterogeneous fluid. When determining the profile of airfoil, the integro-differential equation can be used to calculate the effect of circulation, lift and

${ }^{*}$ Corresponding author.

Emails: xiuli16@email.jlu.edu.cn (X. L. Wang), zhaiql@pku.edu.cn (Q. L. Zhai), zhangran@jlu.edu.cn (R. Zhang), szhang@udel.edu (S. Y. Zhang) 
resistance of the air [10]. Because of their application values, integro-differential equations are essential and significant research subjects. In this paper, we consider the linear parabolic integro-differential equation in domain $\Omega \subset \mathcal{R}^{2}$ with boundary $\partial \Omega$ : seek an unknown function $u=u(\mathbf{x}, t)$ satisfying:

$$
\begin{aligned}
& u_{t}(\mathbf{x}, t)-\nabla \cdot(A \nabla u(\mathbf{x}, t))-\int_{0}^{t} \nabla \cdot(B \nabla u(\mathbf{x}, \zeta)) d \zeta=f(\mathbf{x}, t), \quad(\mathbf{x}, t) \in \Omega \times(0, T], \\
& u=g(\mathbf{x}, t), \\
& (\mathbf{x}, t) \in \partial \Omega \times(0, T], \\
& u(\mathbf{x}, 0)=\psi(\mathbf{x}), \\
& x \in \Omega \text {, }
\end{aligned}
$$

where $\mathbf{x}=\left\{x_{1}, x_{2}\right\}, A=\left[a_{i, j}(\mathbf{x}, t)\right]_{2 \times 2}, B=\left[b_{i, j}(\mathbf{x}, t)\right]_{2 \times 2}, A_{t}=\left[\left(a_{i, j}\right)_{t}(\mathbf{x}, t)\right]_{2 \times 2}$ and $B_{t}=$ $\left[\left(b_{i, j}\right)_{t}(\mathbf{x}, t)\right]_{2 \times 2}$. The matrix-valued functions $A$ and $B$ are sufficiently smooth and $A$ is symmetric. They also satisfy the some properties with positive constants $\alpha_{1}, \alpha_{2}, \alpha_{3}, \beta_{1}, \beta_{2}$ for any $\xi, \eta \in \mathcal{R}^{2}$ that

$$
\begin{array}{ll}
\alpha_{1}\|\xi\|^{2} \leq \xi^{T} A \xi \leq \alpha_{2}\|\xi\|^{2}, & \left\|\xi^{T} A_{t} \eta\right\| \leq \alpha_{3}\|\xi\|\|\eta\|, \\
\left\|\xi^{T} B \eta\right\| \leq \beta_{1}\|\xi\|\|\eta\|, & \left\|\xi^{T} B_{t} \eta\right\| \leq \beta_{2}\|\xi\|\|\eta\| .
\end{array}
$$

Several numerical methods for problem (1.1a)-(1.1c) have been proposed. The earliest ones are the finite element (FE) methods $[2,4,25]$ and the finite volume (FV) methods $[14,16]$. One important characteristic of the finite element method is that it can preserve the conservation of mass and momentum. This method is primarily applied for the diffusion problems and the existence and uniqueness are proved. However, the finite volume method is preferred to the finite element method for conservation and stability. And it is more suitable for the discretization of the conservation of laws. A FV-FE method [12] which combines the advantages of the above methods is proposed. However, these methods require two mutually associated meshes. In order to reduce this correlation, many experts and scholars have proposed various discontinuous Galerkin methods. However, it is difficult to construct the penalty items of the discontinuous Galerkin method $[5,11]$.

Wang and Ye in 2011 proposed the weak Galerkin finite element for the second-order elliptic equations $[19,29]$. The method is applied to many problems, such as Stokes equations [17,18,20-22,28], Brinkman problem [23,27], Biharmonic equations [30], eigenvalue problems [26] and Stochastic problems [6,33] and so on. The partition of the domain can be arbitrary polygonal or polyhedral. The construction of the approximated function is simple and satisfies the stability condition. The essence of the weak Galerkin finite element is that the classical operators are replaced by some weak operators. In paper [32], the weak Galerkin finite element method is applied to the linear parabolic integrodifferential equations. It proposes the semi-discrete and fully-discrete weak Galerkin finite element schemes. The optimal error estimates are obtained.

In this paper, we propose another weak finite element method by adding a stabilizer. The reason why we propose this method is that: firstly, the approximation space is easily constructed and simply satisfies the stability condition; moreover, the element 
of partition can be arbitrary polygon or polyhedron. That means, comparing with the literature [32], the weak Galerkin finite element method with a stabilizer is more effective in dealing with polygon meshes. In particular, polygonal meshes are widely used in the geological fields, such as earthquake prediction, coal mining and petroleum storage, etc.. Therefore, the weak Galerkin finite element method with a stabilizer is more flexible and has a wider range of applications. We also obtain the optimal order convergence in corresponding norms for the semi-discrete scheme and the fully-discrete scheme of the weak Galerkin finite element method, respectively.

The rest of this article is organized as follows. In Section 2, the preparatory work is presented. We propose semi-discrete and fully-discrete weak Galerkin finite element schemes in Section 3. The error estimates in $H^{1}$ norm and $L^{2}$ norm are derived in Section 4. In Section 5, we provide the weak Galerkin finite element method for the primal integro-differential. This part provides further theoretical support for the error estimates in Section 4. Finally, several experiments are presented to verify the validity of above theoretical analysis.

\section{Preparatory work}

In order to construct the variational forms of the weak Galerkin finite element method, we first introduce serval definitions and notations.

In this paper, we use the standard definitions in the Sobolev space $H^{s}(D)$. The associated inner products and norms are denoted by $(\cdot, \cdot)_{s, D}$ and $\|\cdot\|_{s, D}$ with any $s \geq 0$, respectively. The space $H^{0}(D)$ coincides with $L^{2}(D)$. When $D=\Omega$, we shall drop the subscripts $D$ and $s$ in the norm and inner product notation. When $D$ is an edge/face, the $L^{2}$ inner product is represented by $\langle\cdot, \cdot\rangle_{D}$.

It is a well-known fact that the classical variational form of the parabolic integrodifferential equations (1.1a)-(1.1c) is to find $u \in L^{2}\left(0, T ; H^{1}(\Omega)\right)$ satisfying respectively the initial and boundary conditions (1.1b) and (1.1c) with $t \in(0, T]$, such that

$$
\left(u_{t}, v\right)+(A \nabla u, \nabla v)+\int_{0}^{t}(B \nabla u, \nabla v) d \zeta=(f, v), \quad \forall v \in H_{0}^{1}(\Omega) .
$$

Assume that $\mathcal{T}_{h}$ is a partition of the domain $\Omega$, which is a polygons in $\mathbb{R}^{2}$ or polyhedral in $\mathbb{R}^{3}$ and satisfies a set of shape regularity conditions [20]. Denote by $T$ is an any element with $\partial T$ as its boundary. Denote by $\mathcal{E}_{h}$ the set of all edges or faces in $\mathcal{T}_{h}$. For any element $T \in \mathcal{T}_{h}$, denote by $h_{T}$ the diameter of $T$. Similarly, the diameter of $e \in \mathcal{E}_{h}$ is given by $h_{e}$. We define the mesh size of partition $\mathcal{T}_{h}$ as

$$
h=\max _{T \in \mathcal{T}_{h}} h_{T} .
$$

Next, we introduce the weak Galerkin finite element space

$$
\begin{aligned}
& V_{h}=\left\{v=\left\{v_{0}, v_{b}\right\}, v_{0} \in P_{k}(T), v_{b} \in P_{k}(e), \forall T \in \mathcal{T}_{h}, \forall e \in \mathcal{E}_{h}\right\}, \\
& V_{h}^{0}=\left\{v \in V_{h},\left.v_{b}\right|_{\partial \Omega}=0\right\},
\end{aligned}
$$


where $P_{k}(T)$ and $P_{k}(e)$ are the sets of polynomials on $T$ and $e$ with the degree of polynomial no more than $k$. Note that, $v_{0}$ is the internal functions and $v_{b}$ is the boundary functions on each element $T$. There is no relationship between $v_{0}$ and $v_{b}$. We emphasize that $v_{b}$ has a single value on each edge $e \in \mathcal{E}_{h}$.

At last, we define the discrete weak gradient operator. For each $v=\left\{v_{0}, v_{b}\right\} \in V_{h}$, the discrete weak gradient [19] is denoted by $\nabla_{w, k-1} v$ and defined by the following equation

$$
\left(\nabla_{w, k-1} v, \tau\right)=-\left(v_{0}, \nabla \cdot \tau\right)_{T}+\left\langle v_{b}, \tau \cdot n\right\rangle_{\partial T}, \quad \tau \in P_{k-1}(T) .
$$

In the following sections, we will drop the subscript $k-1$ of the discrete weak gradient with the confusion.

\section{Weak Galerkin finite element method}

With the partition of the domain $\Omega$, we define some projections. On each element $T \in \mathcal{T}_{h}$, $Q_{0}$ is the $L^{2}$ projection from $L^{2}(T)$ onto $P_{k}(T)$. For each edge/face $e \in \mathcal{E}_{h}, Q_{b}$ is the $L^{2}$ projection from $L^{2}(e)$ onto $P_{k}(e)$. Combing the projections $Q_{0}$ and $Q_{b}$ are written to $Q_{h}=$ $\left\{Q_{0}, Q_{b}\right\}$. Let $R_{h}$ be a projection from $\left[L^{2}(T)\right]^{d}$ onto $\left[P_{k-1}(T)\right]^{d}$. The projections satisfy the commutative property $\nabla_{w}\left(Q_{h} u\right)=R_{h}(\nabla u)$, which is obtained by the definition of discrete weak gradient and integration by parts [20].

Introduce two bilinear forms with a matrix-valued function $S$ in $\Omega$

$$
\begin{aligned}
& s(w, v)=\sum_{T \in \mathcal{T}_{h}} h_{T}^{-1}\left\langle w_{0}-w_{b}, v_{0}-v_{b}\right\rangle_{\partial T}, \\
& a(S, w, v)=\left(S \nabla_{w} w, \nabla_{w} v\right)_{T}+s(w, v),
\end{aligned}
$$

for all $w, v \in V_{h}$.

From the above preparatory work, we present the semi-discrete weak Galerkin finite element schemes for the linear parabolic integro-differential equations.

Weak Galerkin Algorithm 1. Find $u_{h}(t)=\left\{u_{0}(t), u_{b}(t)\right\} \in L^{2}\left(0, T ; V_{h}\right)$ satisfying

$$
\begin{aligned}
& \left(\left(u_{0}\right)_{t}, v_{0}\right)+a\left(A, u_{h}, v\right)+\int_{0}^{t} a\left(B, u_{h}, v\right) d \zeta=\left(f, v_{0}\right), \\
& u_{b}(t)=Q_{b} g, \\
& u_{h}(0)=E_{h} u(0),
\end{aligned}
$$

for any $v=\left\{v_{0}, v_{b}\right\} \in V_{h}^{0}$.

Let $\tau$ be the step size, $t_{i}=i \tau$ for $i=0,1, \cdots, u_{h}^{i}:=u_{h}\left(t_{i}\right)=\left\{u_{0}^{i}, u_{b}^{i}\right\}$ and $f^{i}:=f\left(t_{i}\right)$. At the time $t=t_{i}$, the backward Euler differential quotient is given by

$$
\delta_{t} u_{h}^{i}=\frac{u_{h}^{i}-u_{h}^{i-1}}{\tau}
$$

With $\delta_{t}$, we construct the fully-discrete weak Galerkin finite element scheme as follows 
Weak Galerkin Algorithm 2. Find $u_{h}^{i}=\left\{u_{0}^{i}, u_{b}^{i}\right\} \in L^{2}\left(0, T ; V_{h}\right)$ with any positive integer $1 \leq i \leq N$ such that

$$
\begin{aligned}
& \left(\delta_{t} u_{0}^{i}, v_{0}\right)+a\left(A^{i}, u_{h}^{i}, v\right)+\tau \sum_{j=0}^{i-1} a\left(B^{j}, u_{h}^{j}, v\right)=\left(f^{i}, v_{0}\right), \\
& u_{b}^{i}=Q_{b} g^{i}, \\
& u_{h}^{0}=E_{h} u^{0},
\end{aligned}
$$

for any $v=\left\{v_{0}, v_{b}\right\} \in V_{h}^{0}$.

Define the norm of space $V_{h}^{0}$ as

$$
\||v|\|^{2}=a(I, v, v),
$$

where $I$ is an identity matrix.

Lemma 3.1. ||$|v|||$ is a norm of weak Galerkin finite element space $V_{h}^{0}$.

Proof. When $\||v|\|=0$, we get $\nabla_{w} v=0$ and $v_{0}=v_{b}$ on $\partial T$. From the fact $\nabla_{w} v=0$ and for any $q \in\left[P_{k-1}(T)\right]^{d}$, we have

$$
\begin{aligned}
0 & =\left(\nabla_{w} v, q\right)_{T} \\
& =-\left(v_{0}, \nabla \cdot q\right)_{T}+\left\langle v_{b}, q \cdot \mathbf{n}\right\rangle_{\partial T} \\
& =\left(\nabla v_{0}, q\right)_{T} .
\end{aligned}
$$

Considering the arbitrariness of $q$ and letting $q=\nabla v_{0}$, we obtain $\left\|\nabla v_{0}\right\|^{2}=0$. That yields $v_{0}=C$ on each element. It follows from $v_{b}=0$ on $\partial \Omega$ that $v_{0}=v_{b}=0$.

Lemma 3.2. For any $v, w \in V_{h}^{0}$, we have

$$
\begin{aligned}
& |a(S, w, v)| \leq C|||w||||||v|||, \\
& |a(A, v, v)| \geq C|||v|||^{2} .
\end{aligned}
$$

Lemma 3.3. For the numerical solution to the semi-discrete weak Galerkin finite element scheme (3.1a) with initial and boundary conditions (3.1b) and (3.1c), there holds that

$$
\left\|u_{h}(t)\right\|^{2} \leq e^{C t}\left(\left\|u_{h}(0)\right\|^{2}+C \int_{0}^{t}\|f(\zeta)\|^{2} d \zeta\right)
$$

Proof. Letting $v=u_{h}$ in the form (3.1a), we have

$$
\left(\left(u_{0}\right)_{t}, u_{0}\right)+a\left(A, u_{h}, u_{h}\right)+\int_{0}^{t} a\left(B, u_{h}, u_{h}\right) d \zeta=\left(f, u_{0}\right) .
$$


It follows from bilinear property of $a(\cdot, \cdot, \cdot)$ that

$$
\left(\left(u_{0}\right)_{t}, u_{0}\right) \leq\left(f, u_{0}\right)
$$

i.e.,

$$
\frac{1}{2} \frac{d}{d t} \int_{\Omega} u_{0}^{2}(t) d x=\left(\left(u_{0}\right)_{t}, u_{0}\right) \leq\left(f, u_{0}\right) \leq C\left(\int_{\Omega} f^{2} d x+\int_{\Omega} u_{0}^{2}(t) d x\right)
$$

Integrating the above inequality with respect to $t$ and using Gronwall lemma, we complete the proof of this lemma.

Theorem 3.1. The solution of the semi-discrete weak Galerkin finite element schemes (3.1a)(3.1c) is unique.

Proof. It is enough to present that the following homogenous equations have a unique zero solution

$$
\begin{array}{lr}
\left(\left(u_{0}\right)_{t}, v\right)+a\left(A, u_{h}, v\right)+\int_{0}^{t} a\left(B, u_{h}, v\right) d \zeta=0, & \forall v \in V_{h}^{0}, \quad 0 \leq t \leq T, \\
u_{b}(t)=0 & \text { on } \partial \Omega, \quad 0 \leq t \leq T, \\
u_{h}(0)=\{0,0\} . &
\end{array}
$$

Taking $v=u_{h}$ in (3.3a), we have

$$
\left(\left(u_{0}\right)_{t}, u_{0}\right)+a\left(A, u_{h}, u_{h}\right)+\int_{0}^{t} a\left(B, u_{h}, u_{h}\right) d \zeta=0 .
$$

Considering the positive definiteness of matrix $A$, the boundedness of the matrix $B$, the Cauchy-Schwarz inequality and the Young's inequality, we get

$$
\begin{aligned}
& \frac{1}{2} \frac{d}{d t}\left(u_{0}, u_{0}\right)=\frac{1}{2} \frac{d}{d t} \int_{\Omega} u_{0}^{2} d x=\left(\left(u_{0}\right)_{t}, u_{0}\right), \\
& a\left(A, u_{h}, u_{h}\right) \geq \alpha_{1}\left\|\left|u_{h}\right|\right\|^{2}, \\
& -\int_{0}^{t} a\left(B, u_{h}(\zeta), u_{h}(t)\right) d \zeta \leq C \int_{0}^{t}\left|\left\|u_{h}(\zeta)\right\|\right|^{2} d \zeta+\left.\frac{\alpha_{1}}{2}\left\|u_{h}\right\|\right|^{2} .
\end{aligned}
$$

It follows from these equations above that

$$
\frac{1}{2} \frac{d}{d t} \int_{\Omega} u_{0}^{2} d x+\frac{\alpha_{1}}{2}\left|\left\|u _ { h } \left|\left\|^{2} \leq C \int_{0}^{t}\left|\left\|u_{h}(\zeta) \mid\right\|^{2} d \zeta .\right.\right.\right.\right.\right.
$$

Integrating above equation from 0 to $t$, we obtain

$$
\frac{1}{2}\left\|u_{0}(t)\right\|^{2}+\frac{\alpha_{1}}{2} \int_{0}^{t}\left\|u_{h}\right\|^{2} d \zeta \leq C \int_{0}^{t} \int_{0}^{\zeta}\left\|u_{h}(\varsigma)\right\|^{2} d \zeta d \zeta
$$


Since $\left\|u_{0}(t)\right\|^{2} \geq 0$, so we have the fact

$$
\frac{\alpha_{1}}{2} \int_{0}^{t}\|\| u_{h}\left\|\left.\right|^{2} d \zeta \leq C \int_{0}^{t} \int_{0}^{\zeta}\right\| u_{h}(\zeta) \|^{2} d \zeta d \zeta
$$

From Gronwall inequality, we get

$$
\int_{0}^{t}\|\| u_{h} \|\left.\right|^{2} d \zeta \leq 0
$$

Considering the property of norm $\left\|\left|u_{h}\right|\right\| \geq 0$, we obtain $u_{h}=0$, which means $u_{0}=u_{b}=0$.

Theorem 3.2. The solution of the fully-discrete weak Galerkin finite element schemes (3.2a)(3.2c) is unique.

Proof. It is analogous to prove the Theorem 3.1. Consider the following homogeneous equations

$$
\begin{array}{ll}
\left(\delta_{t} u_{0}^{i}, v_{0}\right)+a\left(A^{i}, u_{h}^{i}, v\right)+\tau \sum_{j=0}^{i-1} a\left(B^{j}, u_{h}^{j}, v\right)=0, & \forall v \in V_{h}^{0}, \quad 1 \leq i \leq N, \\
u_{b}^{i}=0 & \text { on } \partial \Omega, \quad 1 \leq i \leq N, \\
u_{h}^{0}=\{0,0\} . &
\end{array}
$$

Taking $v=u_{h}^{i}$ in (3.5a), we get

$$
\left(\delta_{t} u_{0}^{i}, u_{0}^{i}\right)+a\left(A^{i}, u_{h}^{i}, u_{h}^{i}\right)+\tau \sum_{j=0}^{i-1} a\left(B^{j}, u_{h}^{j}, u_{h}^{i}\right)=0, \quad 1 \leq i \leq N .
$$

It follows from backward Euler form, the positive definiteness and boundedness of $a(\cdot, \cdot, \cdot)$ and the Young's inequality that

$$
\begin{aligned}
& a\left(A^{i}, u_{h}^{i}, u_{h}^{i}\right) \geq \alpha_{1}\left\|u_{h}^{i}\right\|^{2}, \\
& \left(\delta_{t} u_{0}^{i}, u_{0}^{i}\right)=\frac{1}{2 \tau}\left(\left(u_{0}^{i}, u_{0}^{i}\right)-\left(u_{0}^{i-1}, u_{0}^{i-1}\right)+\left(u_{0}^{i}-u_{0}^{i-1}, u_{0}^{i}-u_{0}^{i-1}\right)\right) \geq \frac{1}{2 \tau}\left(\left\|u_{0}^{i}\right\|^{2}-\left\|u_{0}^{i-1}\right\|^{2}\right),
\end{aligned}
$$

and

$$
-\tau \sum_{j=0}^{i-1} a\left(B^{j}, u_{h}^{j}, u_{h}^{i}\right) \leq\left.\beta_{1} \tau \sum_{j=0}^{i-1}\left\||| u_{h}^{j}\left|\left\|\cdot\left|\left\|u_{h}^{i}\right\|\right| \leq C \beta_{1} \tau \sum_{j=0}^{i-1}\right\|\right| u_{h}^{j}\right\|\right|^{2}+\frac{\alpha_{1}}{2}\left\|\left|u_{h}^{i} \|\right|^{2} .\right.
$$

Summing up all above equations yields

$$
\frac{1}{2 \tau}\left(\left\|u_{0}^{i}\right\|^{2}-\left\|u_{0}^{i-1}\right\|^{2}\right)+\left.\frac{\alpha_{1}}{2}\left\|u_{h}^{i}\right\|\right|^{2} \leq C \beta_{1} \tau \sum_{j=0}^{i-1}\|\| u_{h}^{j}\|\|^{2} .
$$


Accumulating inequality (3.6) with $i$ from 1 to $k-1$ for $1 \leq k \leq N+1$, we obtain

$$
\left\|u_{0}^{k-1}\right\|^{2}+\alpha_{1} \tau \sum_{i=1}^{k-1}\left|\left\|u_{h}^{i}\left|\left\|^{2} \leq C \beta_{1}(\tau)^{2} \sum_{i=1}^{k-1} \sum_{j=1}^{i-1}\right\| u_{h}^{j} \|\right|^{2} .\right.\right.
$$

Considering the discrete Gronwall inequality with the fact $\left\|u_{0}^{k-1}\right\|^{2} \geq 0$, we arrive at

$$
\tau \sum_{i=1}^{k-1}\left|\left\|u_{h}^{i} \mid\right\|^{2} \leq 0, \quad 1 \leq k \leq N+1,\right.
$$

which yields $u_{h}^{i}=0$ with $1 \leq i \leq N$. This theorem is completed.

\section{Error estimates}

In this section, we derive the error equations and error estimates for the semi-discrete and fully-discrete weak Galerkin finite element schemes. First, we define an elliptical projection $E_{h}$, which is similar to Wheeler's projections in [23], for the exact solution $u \in L^{2}\left(0, T ; H^{1}(\Omega)\right)$ of the linear parabolic integro-differential equations (1.1a)-(1.1c). With $E_{h}$ we define an equation with a fixed time $t \in(0, T]$ such that

$$
\begin{aligned}
& a\left(A, E_{h} u, v\right)+\int_{0}^{t} a\left(B, E_{h} u, v\right) d \zeta=(-\nabla \cdot(A \nabla u), v)-\int_{0}^{t}(\nabla \cdot B(\nabla u), v) d \zeta, \\
& E_{b} u=Q_{b} g \quad \text { on } \partial \Omega,
\end{aligned}
$$

for any $v=\left\{v_{0}, v_{b}\right\} \in V_{h}^{0}$.

Denote

$$
\begin{array}{lll}
e=E_{h} u-u_{h}, & \rho=Q_{h} u-E_{h} u, & \eta=u-Q_{h} u, \\
\xi=Q_{0} u_{t}-\delta_{t}\left(Q_{0} u\right), & \rho_{t}=Q_{h} u_{t}-E_{h} u_{t}, & \eta_{t}=u_{t}-Q_{h} u_{t} .
\end{array}
$$

\subsection{Semi-discrete WG error estimates}

Firstly, we derive the semi-discrete error equations. Then, we use the error equations to derive the semi-discrete error estimates in $H^{1}$ norm and $L^{2}$ norm.

Lemma 4.1. Assume $u_{h} \in L^{2}\left(0, T ; V_{h}\right)$ is the numerical solution of semi-discrete WG schemes (3.1a)-(3.1c) and $u \in L^{2}\left(0, T ; H^{1}(\Omega)\right)$ is the exact solution of linear parabolic integro-differential equations (1.1a)-(1.1c) for $0<t \leq T$. Then, for any $v \in V_{h}^{0}$, we have

$$
\left(\left(e_{0}\right)_{t}, v_{0}\right)+a(A, e, v)+\int_{0}^{t} a(B, e, v) d \zeta=-\left(\left(\rho_{0}\right)_{t}, v_{0}\right) .
$$


Proof. Testing the integral differential equation (1.1a) by $v=\left\{v_{0}, v_{b}\right\} \in V_{h}^{0}$ and considering the definition of projection $E_{h}$, we get

$$
\left(u_{t}, v\right)+a\left(A, E_{h} u, v\right)+\int_{0}^{t} a\left(B, E_{h} u, v\right) d \zeta=\left(f, v_{0}\right) .
$$

It follows from the semi-discrete variational equation (3.1a) that

$$
\left(E_{0} u_{t}-\left(u_{0}\right)_{t}, v_{0}\right)+a\left(A, E_{h} u-u_{h}, v\right)+\int_{0}^{t} a\left(B, E_{h} u-u, v\right) d \zeta=-\left(Q_{0} u_{t}-E_{0} u_{t}, v_{0}\right) .
$$

Following the definition of $e$ and $\rho$, we obtain the semi-discrete error equation.

Theorem 4.1. Assume $u \in L^{2}\left(0, T ; H^{s+1}(\Omega)\right)$ and $u_{h} \in L^{2}\left(0, T ; V_{h}\right)$ are the solutions of integrodifferential equations (1.1a)-(1.1c) and semi-discrete weak Galerkin finite element variational forms (3.1a)-(3.1c), respectively. There exists a positive constant $C$ independent of $h$ satisfying

$$
\begin{aligned}
& \|e\|^{2} \leq C h^{2(s+1)} \int_{0}^{t}\left(\|u\|_{s+1}^{2}+\left\|u_{t}\right\|_{s+1}^{2}\right) d \zeta, \\
& \|\| e \|^{2} \leq C h^{2(s+1)} \int_{0}^{t}\left(\|u\|_{s+1}^{2}+\left\|u_{t}\right\|_{s+1}^{2}\right) d \zeta .
\end{aligned}
$$

Proof. Firstly, taking $v=e$ in the semi-discrete error equation (4.1), we have

$$
\left(\left(e_{0}\right)_{t}, e_{0}\right)+a(A, e, e)=-\left(\left(\rho_{0}\right)_{t}, v_{0}\right)-\int_{0}^{t} a(B, e, e) d \zeta .
$$

It follows from property of $a(\cdot, \cdot, \cdot)$, the Cauchy-Swarch inequality and the Young's inequality that

$$
\begin{array}{ll}
\left(\left(e_{0}\right)_{t}, e_{0}\right)=\frac{1}{2} \frac{d}{d t}\left(e_{0}, e_{0}\right)=\frac{1}{2}\left\|e_{0}\right\|^{2}, \quad & a(A, e, e) \geq \alpha_{1}\|\| \|^{2}, \\
-\int_{0}^{t} a(B, e, e) \leq \int_{0}^{t} \beta_{1}\|e \mid\|^{2} d \zeta, & -\left(\left(\rho_{0}\right)_{t}, e_{0}\right) \leq C\left\|\left(\rho_{0}\right)_{t}\right\|^{2}+\frac{1}{2}\left\|e_{0}\right\|^{2},
\end{array}
$$

which leads to

$$
\frac{d}{d t}\left\|e_{0}\right\|^{2}+\left\|\left|\left\|^{2} \leq C \int_{0}^{t}\right\|\right|\right\| \|^{2} d \zeta+C\left(\left\|\left(\rho_{0}\right)_{t}\right\|^{2}+\left\|e_{0}\right\|^{2}\right)
$$

Integrating above equation from 0 to $t$ with the fact $e(\cdot, 0)=0$ yields

$$
\left\|e_{0}\right\|^{2}+\int_{0}^{t}\|\| e\|\|^{2} d \zeta \leq C \int_{0}^{t}\left(\int_{0}^{\tau}\|e\|^{2} d \zeta+\left\|e_{0}\right\|^{2}\right) d \zeta+C \int_{0}^{t}\left\|\left(\rho_{0}\right)_{t}\right\|^{2} d \zeta .
$$

By the Gronwall's inequality and the estimate of $\left\|\left(\rho_{0}\right)_{t}\right\|$ in the Theorem 5.3, we obtain an error estimate formula (4.3a). 
Next, for each fixed $t$, letting $v=e_{t}$ in semi-discrete error equation (4.1) yields

$$
\left(\left(e_{0}\right)_{t},\left(e_{0}\right)_{t}\right)+a\left(A, e, e_{t}\right)=-\int_{0}^{t} a\left(B, e, e_{t}\right) d \zeta-\left(\left(\rho_{0}\right)_{t},\left(e_{0}\right)_{t}\right) .
$$

Since $\frac{d}{d t} a(S, e, e)=a\left(S_{t}, e, e\right)+2 a\left(S, e, e_{t}\right)$ with $S \in\{A, B\}$, we obtain

$$
\begin{aligned}
& \left\|\left(e_{0}\right)_{t}\right\|^{2}+\frac{1}{2} \frac{d}{d t} a(A, e, e) \\
= & \frac{1}{2} a\left(A_{t}, e, e\right)-\frac{1}{2} a(B, e, e)+\frac{1}{2} \int_{0}^{t} a\left(B_{t}, e, e\right) d \zeta-\left(\left(\rho_{0}\right)_{t},\left(e_{0}\right)_{t}\right) .
\end{aligned}
$$

Using the definition of $\||\cdot|\|$, the property of $A$ and $B$ and the Young's inequality, we obtain

$$
\begin{array}{ll}
a(A, e, e) \geq \alpha_{1}\left\|\left|\|\mid\|^{2},\right.\right. & a\left(A_{t}, e, e\right) \leq \alpha_{3}\|\mid e\|^{2}, \\
-a(B, e, e) \leq \beta_{1}\|\| \|^{2}, & a\left(B_{t}, e, e\right) \leq \beta_{2}\|e\|^{2},
\end{array}
$$

and

$$
-\left(\left(\rho_{0}\right)_{t},\left(e_{0}\right)_{t}\right) \leq \frac{1}{4}\left\|\left(\rho_{0}\right)_{t}\right\|^{2}+\left\|\left(e_{0}\right)_{t}\right\|^{2} .
$$

Substituting all these forms into (4.4) yields

$$
\frac{1}{2} \frac{d}{d t}\|\| e\|\|^{2} \leq C\left(\|||\|^{2}+\left\|\left(\rho_{0}\right)_{t}\right\|^{2}\right)+C \int_{0}^{t}\|\| e\|\|^{2} d \zeta .
$$

Integrating the above formula with respect to $t$, we have

$$
\|\mid\|^{2} \leq C \int_{0}^{t}\|\|\left\|^{2} d \zeta+C \int_{0}^{t}\right\|\left(\rho_{0}\right)_{t} \|^{2} d \zeta .
$$

From Gronwall inequality and the estimate of $\left\|\left(\rho_{0}\right)_{t}\right\|$, we obtain an error estimate formula (4.3b).

\subsection{Fully-discrete WG error estimates}

Firstly, we derive the fully discrete error equations. Then, we use the error equations to derive the fully-discrete error estimates in $H^{1}$ norm and $L^{2}$ norm.

Lemma 4.2. Let $u_{h} \in L^{2}\left(0, T ; V_{h}\right)$ be the numerical solution of fully-discrete WG schemes (3.2a)(3.2c) and $u \in L^{2}\left(0, T ; H^{1}(\Omega)\right)$ be the exact solution of linear parabolic integro-differential equations (1.1a)-1.1c with $0<t \leq T$. Then, for any $v \in V_{h}^{0}$ and $1 \leq i \leq N$, we have

$$
\left(\delta_{t} e_{0}^{i}, v_{0}\right)+a\left(A^{i}, e^{i}, v\right)=-\left(\xi_{0}^{i}, v_{0}\right)-\left(\delta_{t} \rho_{0}^{i}, v_{0}\right)-\int_{0}^{t^{i}} a\left(B, E_{h} u, v\right) d \zeta+\tau \sum_{j=0}^{i-1} a\left(B^{j}, u_{h}^{j}, v\right) .
$$


Proof. Using $v=\left\{v_{0}, v_{b}\right\} \in V_{h}^{0}$ to test the integro-differential equation (1.1a) and the definition of $E_{h}$, we have

$$
\left(Q_{0} u_{t}, v_{0}\right)+a\left(A, E_{h} u, v\right)+\int_{0}^{t} a\left(B, E_{h} u, v\right) d \zeta=\left(f, v_{0}\right) .
$$

Considering the fully-discrete variational forms and the equation above with $t=t^{i}$ and $1 \leq i \leq N$, we obtain

$$
\left(\delta_{t} e_{0}^{i}, v_{0}\right)+a\left(A^{i}, e^{i}, v\right)=-\left(\xi_{0}^{i}, v_{0}\right)-\left(\delta_{t} \rho_{0}^{i}, v_{0}\right)-\int_{0}^{t^{i}} a\left(B, E_{h} u, v\right) d \zeta+\tau \sum_{j=0}^{i-1} a\left(B^{j}, u_{h^{j}}^{j} v\right) .
$$

Thus, we complete the proof.

Similarly, following the error estimates of semi-discrete WG schemes, we construct the error estimates of fully-discrete WG schemes in $H^{1}$ norm and $L^{2}$ norm, respectively.

Theorem 4.2. Let $u_{h}^{n} \in L^{2}\left(0, T ; V_{h}\right)$ be the solution of the problems (5.3a)-(5.3b) arising from fully-discrete weak Galerkin finite element schemes (3.2a)-(3.2c). Assume $u \in L^{2}\left(0, T ; H^{s+1}(\Omega)\right.$ ) is the exact solution of integro-differential problems (5.3a)-(5.3b). Then, for any $v \in V_{h}^{0}, 1 \leq k \leq N$ and $n=0,1 \cdots$, we obtain

$$
\begin{gathered}
\left\|e_{0}^{k}\right\|^{2} \leq C \\
\tau^{2}\left(\left\|u_{t t}\right\|_{L^{2}\left(0, T ; L^{2}\right)}^{2}+\|u\|_{L^{2}\left(0, T ; H^{s+1}\right)}^{2}+\left\|u_{t}\right\|_{L^{2}\left(0, T ; H^{s+1}\right)}^{2}\right) \\
+C h^{2(s+1)}\left(\|u\|_{L^{2}\left(0, T ; H^{s+1}\right)}^{2}+\left\|u_{t}\right\|_{L^{2}\left(0, T ; H^{s+1}\right)}^{2}\right) .
\end{gathered}
$$

Proof. Taking $v=e^{i}$ in Lemma 4.2 for the fully-discrete error equation, we have

$$
\begin{aligned}
& \left(\delta_{t} e_{0}^{i}, e_{0}^{i}\right)+a\left(A^{i}, e^{i}, e^{i}\right) \\
=- & \left(\eta_{0}^{i}, e_{0}^{i}\right)-\left(\delta_{t} \rho_{0}^{i}, e_{0}^{i}\right)-\int_{0}^{t^{i}} a\left(B, E_{h} u, e^{i}\right) d \zeta+\tau \sum_{j=0}^{i-1} a\left(B^{j}, E_{h} u\left(t^{j}\right), e^{i}\right) \\
& -\tau \sum_{j=0}^{i-1} a\left(B^{j}, E_{h} u\left(t_{j}\right)-u_{h}^{j}, e^{i}\right), \quad 1 \leq i \leq N .
\end{aligned}
$$

It follows from the backward Euler form, the definition of $\||\cdot|||$ and the Young's inequality that

$$
\begin{aligned}
& \left|\left(\delta_{t} e_{0}^{i}, e_{0}^{i}\right)\right|=\frac{1}{2 \tau}\left(\left(e_{0}^{i}, e_{0}^{i}\right)-\left(e_{0}^{i-1}, e_{0}^{i-1}\right)+\left(e_{0}^{i}-e_{0}^{i-1}, e_{0}^{i}-e_{0}^{i-1}\right)\right) \geq \frac{1}{2 \tau}\left(\left\|e_{0}^{i}\right\|^{2}-\left\|e_{0}^{i-1}\right\|^{2}\right), \\
& \left|a\left(A^{i}, e^{i}, e^{i}\right)\right| \geq \alpha_{1}\left\|\left|e^{i} \|\right|^{2} .\right.
\end{aligned}
$$

Notice that

$$
\xi_{0}^{i}=Q_{0} u_{t}^{i}-\delta_{t}\left(Q_{0} u^{i}\right)=Q_{0}\left(u_{t}^{i}-\delta_{t} u^{i}\right)=Q_{0}\left(\frac{1}{\tau} \int_{t^{i-1}}^{t^{i}}\left(\zeta-t^{i-1}\right) u_{t t}(\zeta) d \zeta\right),
$$


we obtain

$$
\left\|\xi_{0}^{i}\right\| \leq C\left\|\int_{t^{i-1}}^{t^{i}}\left|u_{t t}(\zeta)\right| d \zeta\right\| \leq C\left(\tau \int_{t^{i-1}}^{t^{i}}\left\|u_{t t}\right\|^{2} d \zeta\right)^{\frac{1}{2}}
$$

So, we have

$$
\left|\left(\xi_{0}^{i}, e_{0}^{i}\right)\right| \leq C\left\|\xi_{0}^{i}\right\|^{2}+\frac{1}{4}\left\|e_{0}^{i}\right\|^{2} \leq C \tau \int_{t^{i-1}}^{t^{i}}\left\|u_{t t}\right\|^{2} d \zeta+\frac{1}{4}\left\|e_{0}^{i}\right\|^{2}
$$

Thanks to

$$
\left\|\delta_{t} \rho_{0}^{i}\right\|^{2}=\left\|\frac{\rho_{0}^{i}-\rho_{0}^{i-1}}{\tau}\right\|^{2}=\frac{1}{\tau^{2}}\left\|\int_{t^{i-1}}^{t^{i}}\left(\rho_{0}\right)_{t} d \zeta\right\|^{2} \leq \frac{1}{\tau} \int_{t^{i-1}}^{t^{i}}\left\|\left(\rho_{0}\right)_{t}\right\|^{2} d \zeta
$$

we get

$$
\left|-\left(\delta_{t} \rho_{0}^{i}, e_{0}^{i}\right)\right| \leq \frac{C}{\tau} \int_{t^{i-1}}^{t^{i}}\left\|\left(\rho_{0}\right)_{t}\right\|^{2} d \zeta+\frac{1}{4}\left\|e_{0}^{i}\right\|^{2}
$$

Since

$$
\frac{d}{d t}\left(B \nabla_{w}\left(E_{h} u\right)\right)=B_{t} \nabla_{w}\left(E_{h} u\right)+B \nabla_{w}\left(E_{h} u_{t}\right),
$$

we obtain

$$
\begin{aligned}
& \quad\left|\int_{0}^{t^{i}} a\left(B, E_{h} u, e^{i}\right) d \zeta-\tau \sum_{j=0}^{i-1} a\left(B^{j}, E_{h} u\left(t^{j}\right), e^{i}\right)\right| \\
& =\int_{0}^{t^{i}} s\left(E_{h} u, e^{i}\right) d \zeta-\tau \sum_{j=0}^{i-1} s\left(E_{h} u\left(t^{j}\right), e^{i}\right) \\
& \quad+\left(\sum_{j=0}^{i-1} \int_{t^{j}}^{t^{j+1}}\left[B \nabla_{w}\left(E_{h} u(\zeta)\right)-B^{j} \nabla_{w}\left(E_{h} u\left(t^{j}\right)\right)\right] d \zeta, \nabla_{w} e^{i}\right) .
\end{aligned}
$$

We have the fact

$$
\begin{aligned}
& \left|\left(\sum_{j=0}^{i-1} \int_{t^{j}}^{t^{j+1}}\left[B \nabla_{w}\left(E_{h} u(\zeta)\right)-B^{j} \nabla_{w}\left(E_{h} u\left(t^{j}\right)\right)\right] d \zeta, \nabla_{w} e^{i}\right)\right| \\
= & \left(\sum_{j=0}^{i-1} \int_{t^{j}}^{t^{j+1}} \int_{t^{j}}^{\zeta} \frac{d}{d \zeta}\left[B \nabla_{w}\left(E_{h} u(\zeta)\right)\right] d \zeta d \zeta, \nabla_{w} e^{i}\right) \\
= & \left(\sum_{j=0}^{i-1} \int_{t^{j}}^{t^{j+1}} \int_{t^{j}}^{\zeta}\left[B_{t} \nabla_{w}\left(E_{h}(\zeta)\right)+B \nabla_{w}\left(E_{h} u_{t}(\zeta)\right)\right] d \zeta d \zeta, \nabla_{w} e^{i}\right) \\
\leq & \left(\sum_{j=0}^{i-1} \Delta t \int_{t^{j}}^{t^{j+1}}\left[B_{t} \nabla_{w}\left(E_{h}(\zeta)\right)+B \nabla_{w}\left(E_{h} u_{t}(\zeta)\right)\right] d \zeta, \nabla_{w} e^{i}\right)
\end{aligned}
$$




$$
\begin{aligned}
& =\left(\tau \int_{0}^{t^{i}}\left[B_{t} \nabla_{w}\left(E_{h}(\zeta)\right)+B \nabla_{w}\left(E_{h} u_{t}(\zeta)\right)\right] d \zeta, \nabla_{w} e^{i}\right) \\
& \leq C \tau^{2}\left(\|u\|_{L^{\infty}\left(0, T ; H^{s+1}\right)}^{2}+\left\|u_{t}\right\|_{L^{\infty}\left(0, T ; H^{s+1}\right)}^{2}\right)+\frac{\alpha_{1}}{6}\left\|e^{i}\right\| \|^{2},
\end{aligned}
$$

and

$$
\begin{aligned}
& \left|\int_{0}^{t^{i}} s\left(E_{h} u, e^{i}\right) d \zeta-\tau \sum_{j=0}^{i-1} s\left(E_{h} u\left(t^{j}\right), e^{i}\right)\right| \\
= & \sum_{j=0}^{i-1} \int_{t^{j}}^{t^{j+1}} s\left(E_{h} u, e^{i}\right) d \zeta-\sum_{j=0}^{i-1} \int_{t^{j}}^{t^{j+1}} s\left(E_{h} u\left(t^{j}\right), e^{i}\right) d \zeta \\
= & \sum_{j=0}^{i-1} \int_{t^{j}}^{t^{j+1}} s\left(E_{h} u-E_{h} u\left(t^{j}\right), e^{i}\right) d \zeta \\
= & \sum_{j=0}^{i-1} \int_{t^{j}}^{t^{j+1}} \sum_{T \in \mathcal{T}_{h}} h_{T}^{-1}\left\langle\left(E_{0} u-E_{b} u\right)-\left(E_{0} u\left(t^{j}\right)-E_{b} u\left(t^{j}\right)\right), e_{0}^{i}-e_{b}^{i}\right\rangle_{\partial T} d \zeta \\
\leq & \sum_{T \in \mathcal{T}_{h}}\left\langle\sum_{j=0}^{i-1} \tau \int_{t^{j}}^{t^{j+1}} h_{T}^{-1}\left[E_{0} u_{t}(\zeta)-E_{b} u_{t}(\zeta)\right] d \zeta, e_{0}^{i}-e_{b}^{i}\right\rangle_{\partial T} \\
\leq & \sum_{T \in \mathcal{T}_{h}} \tau\left\langle\int_{0}^{t^{i}} h_{T}^{-1}\left[E_{0} u_{t}(\zeta)-E_{b} u_{t}(\zeta)\right] d \zeta, e_{0}^{i}-e_{b}^{i}\right\rangle_{\partial T} \\
\leq & C \tau^{2}\left\|u_{t}\right\|_{L^{\infty}\left(0, T ; H^{s+1}\right.}^{2}+\left.\frac{\alpha_{1}}{6}\left\|e^{i}\right\|\right|^{2} .
\end{aligned}
$$

From the property of $a(\cdot, \cdot, \cdot)$, we get

$$
\begin{aligned}
& \tau \sum_{j=0}^{i-1} a\left(B^{j}, E_{h} u\left(t^{j}\right)-u_{h}^{j}, e^{i}\right) \leq \beta_{1} \tau \sum_{j=0}^{i-1}\left\|E_{h} u\left(t^{j}\right)-u_{h}^{j}\right\|\|\| e^{i} \| \mid \\
\leq & \left.C \tau^{2} \sum_{j=0}^{j-1}\left\|e^{j}\right\|\right|^{2}+\left.\frac{\alpha_{1}}{6}\left\|e^{i}\right\|\right|^{2} .
\end{aligned}
$$

Combing all the equations above, we get

$$
\begin{aligned}
& \quad\left\|e_{0}^{i}\right\|^{2}-\left\|e_{0}^{i-1}\right\|^{2}+2 \alpha_{1} \tau \mid\left\|e^{i}\right\| \|^{2} \\
& \leq C \tau^{2} \int_{t^{i-1}}^{t^{i}}\left\|u_{t t}\right\|^{2} d \zeta+\tau\left\|e_{0}^{i}\right\|^{2}+C \int_{t^{i-1}}^{t^{i}}\left\|\left(\rho_{0}\right)_{t}\right\|^{2} d \zeta \\
& \quad+C \zeta^{3}\left(\|u\|_{L^{\infty}\left(0, T ; H^{s+1}\right)}^{2}+\left\|u_{t}\right\|_{L^{\infty}\left(0, T ; H^{s+1}\right)}^{2}\right)+\alpha_{1} \tau\left\|e^{i}\right\|\left\|^{2}+C \tau^{3} \sum_{j=0}^{j-1}\right\| e^{j}\|\|^{2} .
\end{aligned}
$$


Accumulating the above equation with $i$ from 1 to $k-1$, we obtain

$$
\begin{aligned}
& \left\|e_{0}^{k-1}\right\|^{2}+\alpha_{1} \tau \sum_{i=1}^{k-1}\|\mid\| \|^{2} \\
& \leq C \tau^{2} \int_{0}^{t^{k-1}}\left\|u_{t t}\right\|^{2} d \zeta+\tau \sum_{i=1}^{k-1}\left\|e_{0}^{i}\right\|^{2}+C \int_{0}^{t^{k}}\left\|\left(\rho_{0}\right) t\right\|^{2} d \zeta \\
& \quad+C \tau^{2}\left(\|u\|_{L^{\infty}\left(0, T ; H^{s+1}\right)}^{2}+\left\|u_{t}\right\|_{L^{\infty}\left(0, T ; H^{s+1}\right)}^{2}\right)+C \tau^{3} \sum_{i=1}^{k-1} \sum_{j=1}^{i-1}\left\|e^{j}\right\| \|^{2},
\end{aligned}
$$

where $1 \leq k \leq N+1$. Following the Gronwall inequality and the estimates of $\|\rho\| \|$, we obtain the fully-discrete error estimate.

Theorem 4.3. Let $u_{h}^{n} \in V_{h}$ be the solution of the problems (5.3a)-(5.3b) arising from fully-discrete weak Galerkin finite element schemes (3.2a)-(3.2c). Assume $u \in L^{2}\left(0, T ; H^{s+1}(\Omega)\right)$ is the exact solution of (5.3a)-(5.3b). Then, for any $v \in V_{h}, 1 \leq k \leq N$ and $n=0,1 \cdots$, we have

$$
\begin{aligned}
\|\| e^{k}\|\|^{2} \leq C \tau^{2} & \left(\left\|u_{t t}\right\|_{L^{2}\left(0, T ; H^{s+1}\right)}^{2}+\left\|u_{t}\right\|_{L^{\infty}\left(0, T ; L^{2}\right)}^{2}+\|u\|_{L^{\infty}\left(0, T ; H^{s+1}\right)}^{2}\right) \\
& +C h^{2(s+1)}\left(\left\|u_{t}\right\|_{L^{2}\left(0, T ; H^{s+1}\right)}^{2}+\|u\|_{L^{2}\left(0, T ; H^{s+1}\right)}^{2}\right) .
\end{aligned}
$$

Proof. Letting $v=\delta_{t} e^{i}$ in the fully-discrete error equation in Lemma 4.2, we obtain

$$
\begin{gathered}
\left\|\delta_{t} e_{0}^{i}\right\|^{2}+a\left(A^{i}, e^{i}, \delta_{t} e^{i}\right) \\
=-\left(\xi_{0}^{i}, \delta_{t} e_{0}^{i}\right)-\left(\delta_{t} \rho_{0}^{i}, \delta_{t} e_{0}^{i}\right)-\int_{0}^{t^{i}} a\left(B, E_{h} u, \delta_{t} e^{i}\right) d \zeta+\tau \sum_{i_{0}=0}^{i-1} a\left(B^{i_{0}}, u_{h}^{i_{0}}, \delta_{t} e^{i}\right) \\
=-\left(\xi_{0}^{i}, \delta_{t} e_{0}^{i}\right)-\left(\delta_{t} \rho_{0}^{i}, \delta_{t} e_{0}^{i}\right)+\delta_{t}\left(\int_{0}^{t^{i}} a\left(B, E_{h} u, e^{i}\right) d \zeta-\tau \sum_{j=0}^{i-1} a\left(B^{j}, u_{h}^{j}, e^{i}\right)\right) \\
\quad-\frac{1}{\tau}\left(\int_{t^{i-1}}^{t^{i}} a\left(B, E_{h} u, e^{i-1}\right) d \zeta-\tau a\left(B^{i-1}, E_{h} u\left(t^{i-1}\right), e^{i-1}\right)\right)-a\left(B^{i-1}, e^{i-1}, e^{i-1}\right) .
\end{gathered}
$$

We estimate the second term and the right hand side terms as follows

$$
\begin{aligned}
& a\left(A^{i}, e^{i}, \delta_{t} e^{i}\right) \geq \frac{1}{2 \tau}\left(a\left(A^{i}, e^{i}, e^{i}\right)-a\left(A^{i}, e^{i}, e^{i-1}\right)\right), \\
& -\left(\xi_{0}^{i}, \delta_{t} e_{0}^{i}\right) \mid \leq C\left(\left\|\xi_{0}^{i}\right\|^{2}+\frac{1}{4}\left\|\delta_{t} e_{0}^{i}\right\|^{2}\right), \\
& \left|-\left(\delta_{t} \rho_{0}^{i}, \delta_{t} e_{0}^{i}\right)\right| \leq C\left\|\delta_{t} \rho_{0}^{i}\right\|^{2}+\frac{1}{4}\left\|\delta_{t} e_{0}^{i}\right\|^{2}, \\
& \left|a\left(B^{i-1}, e^{i-1}, e^{i-1}\right)\right| \leq C\left\|\left|e^{i-1}\right|\right\|^{2} .
\end{aligned}
$$


Notice that

$$
\begin{aligned}
& \left|\frac{1}{\tau}\left(\int_{t^{i-1}}^{t^{i}} a\left(B, E_{h} u, e^{i-1}\right) d \zeta-\tau a\left(B^{i-1}, E_{h} u\left(t^{i-1}\right), e^{i-1}\right)\right)\right| \\
=\mid & \frac{1}{\tau}\left(\int_{t^{i-1}}^{t^{i}}\left(B \nabla_{w}\left(E_{h} u\right), \nabla_{w} e^{i-1}\right) d \zeta-\tau\left(B^{i-1} \nabla_{w}\left(E_{h} u\left(t^{i-1}\right)\right), \nabla_{w} e^{i-1}\right)\right) \mid \\
& +\left|\frac{1}{\tau}\left(\int_{t^{i-1}}^{t^{i}} s\left(E_{h} u, e^{i-1}\right) d \zeta-\tau s\left(E_{h} u, e^{i-1}\right)\right)\right|,
\end{aligned}
$$

where

$$
\begin{aligned}
& \left|\frac{1}{\tau}\left(\int_{t^{i-1}}^{t^{i}}\left(B \nabla_{w}\left(E_{h} u\right), \nabla_{w} e^{i-1}\right) d \zeta-\tau\left(B^{i-1} \nabla_{w}\left(E_{h} u\left(t^{i-1}\right)\right), \nabla_{w} e^{i-1}\right)\right)\right| \\
= & \left.\mid\left(\frac{1}{\tau} \int_{t^{i-1}}^{t^{i}}\left(B \nabla_{w}\left(E_{h} u\right)-B^{i-1} \nabla_{w}\left(E_{h} u\left(t^{i-1}\right)\right)\right) d \zeta, \nabla_{w} e^{i-1}\right)\right) \mid \\
= & \left|\left(\frac{1}{\tau} \int_{t^{i-1}}^{t^{i}} \int_{t^{i-1}}^{\zeta} \frac{d}{d \zeta}\left(B \nabla_{w}\left(E_{h} u(\zeta)\right)\right) d \zeta d \zeta, \nabla_{w} e^{i-1}\right)\right| \\
= & \left|\left(\frac{1}{\tau} \int_{t^{i-1}}^{t^{i}} \int_{t^{i-1}}^{\zeta}\left(B_{t} \nabla_{w}\left(E_{h} u\right)+B \nabla_{w}\left(E_{h} u_{t}\right)\right) d \zeta d \zeta, \nabla_{w} e^{i-1}\right)\right| \\
\leq & \left.C \tau\left(\beta_{1}\|u\|_{L^{\infty}\left(0, T ; H^{s+1}\right)}^{2}+\beta_{2}\left\|u_{t}\right\|_{L^{\infty}\left(0, T ; H^{s+1}\right)}^{2}\right)\right)^{\frac{1}{2}}\left|\left\|e^{i-1}|\||\right.\right. \\
\leq & C \tau^{2}\left(\beta_{1}\|u\|_{L^{\infty}\left(0, T ; H^{s+1}\right)}^{2}+\beta_{2}\left\|u_{t}\right\|_{L^{\infty}\left(0, T ; H^{s+1}\right)}^{2}\right)+\left\|e^{i-1} \mid\right\|^{2},
\end{aligned}
$$

and

$$
\begin{aligned}
& \left|\frac{1}{\tau}\left(\int_{t^{i-1}}^{t^{i}} s\left(E_{h} u, e^{i-1}\right) d \zeta-\tau s\left(E_{h} u, e^{i-1}\right)\right)\right| \\
= & \left|\frac{1}{\tau} \int_{t^{i-1}}^{t^{i}} s\left(E_{h} u-E_{h} u\left(t^{i-1}\right), e^{i-1}\right) d \zeta\right| \\
= & \left|\frac{1}{\tau} \int_{t^{i-1}}^{t^{i}} \sum_{T \in \mathcal{T}_{h}} h_{T}^{-1}\left\langle E_{0} u-E_{b} u-\left(E_{0} u\left(t^{i-1}\right)-E_{b} u\left(t^{i-1}\right)\right), e_{0}^{i-1}-e_{b}^{i-1}\right\rangle_{\partial T} d \zeta\right| \\
\leq & \left|\sum_{T \in \mathcal{T}_{h}}\left\langle\int_{t^{i-1}}^{t^{i}} h_{T}^{-1}\left(E_{0} u_{t}(\zeta)-E_{b} u_{t}(\zeta)\right) d \zeta, e_{0}^{i-1}-e_{b}^{i-1}\right\rangle_{\partial T}\right| \\
\leq & C \tau^{2}\|u\|_{L^{\infty}\left(0, T ; H^{s+1}\right)}^{2}+\left|\left\|e^{i-1} \mid\right\|^{2} .\right.
\end{aligned}
$$


Summing the equations above, we get

$$
\begin{aligned}
& \tau\left\|\delta_{t} e_{0}^{i}\right\|^{2}+a\left(A^{i}, e^{i}, e^{i}\right)-a\left(A^{i}, e^{i}, e^{i-1}\right) \\
& \leq C \tau^{2} \int_{t^{i-1}}^{t^{i}}\left\|u_{t}\right\|^{2} d \zeta+C \int_{t^{i-1}}^{t^{i}}\left\|\left(\rho_{0}\right)_{t}\right\|^{2} d \zeta+C \tau\|\| e^{i-1} \|^{2} \\
& \quad+C \tau^{3}\left(\beta_{1}\|u\|_{L^{\infty}\left(0, T ; H^{s+1}\right)}^{2}+\beta_{2}\left\|u_{t}\right\|_{L^{\infty}\left(0, T ; H^{s+1}\right)}^{2}\right) \\
& \quad+2 \tau \delta_{t}\left(\int_{0}^{t^{i}} a\left(B, E_{h} u, e^{i}\right) d \zeta-\tau \sum_{j=0}^{i-1} a\left(B^{j}, u_{h^{j}}^{j} \delta_{t} e^{i}\right)\right) .
\end{aligned}
$$

Adding the above with respect to $i$ from 1 to $k$ with $e_{0}^{0}=0$ and $1 \leq k \leq N$, we have

$$
\begin{aligned}
\left.\left\|e^{k}\right\|\right|^{2} \leq C & \tau^{2} \int_{0}^{t^{k}}\left\|u_{t t}\right\|^{2} d \zeta+C \int_{0}^{t^{k}}\left\|\left(\rho_{0}\right)_{t}\right\|^{2} d \zeta+C \tau \sum_{i=1}^{k}\left\|e^{i}\right\| \|^{2} \\
& +C \tau^{2}\left(\|u\|_{L^{\infty}\left(0, T ; H^{s+1}\right)}^{2}+\left\|u_{t}\right\|_{L^{\infty}\left(0, T ; H^{s+1}\right)}^{2}\right) \\
& -2\left(\int_{0}^{t^{k}} a\left(B, E_{h} u, e^{k}\right) d \zeta-\tau \sum_{j=0}^{k-1} a\left(B^{j}, u_{h}^{j}, e^{k}\right)\right) .
\end{aligned}
$$

It follows from the proof of the estimates of $\left\|e_{0}^{k}\right\|$ that

$$
\begin{aligned}
& \int_{0}^{t^{k}} a\left(B, E_{h} u, e^{k}\right) d \zeta-\tau \sum_{j=0}^{k-1} a\left(B^{j}, u_{h^{j}}^{j} e^{k}\right) \\
\leq & C \tau^{2}\left(\|u\|_{L^{\infty}\left(0, T ; H^{s+1}\right)}^{2}+\left\|u_{t}\right\|_{L^{\infty}\left(0, T ; H^{s+1}\right)}^{2}\right)+\left.\frac{1}{2}\left\|e^{k}\right\|\right|^{2} .
\end{aligned}
$$

Using the Gronwall inequality and the estimate of $\left\|\left(\rho_{0}\right)_{t}\right\|$, we have

$$
\begin{gathered}
\|\| e^{k} \mid \|^{2} \leq C \tau^{2}\left(\left\|u_{t t}\right\|_{L^{2}\left(0, T ; H^{s+1}\right)}^{2}+\left\|u_{t}\right\|_{L^{\infty}\left(0, T ; L^{2}\right)}^{2}+\|u\|_{L^{\infty}\left(0, T ; H^{s+1}\right)}^{2}\right) \\
+C h^{2(s+1)}\left(\left\|u_{t}\right\|_{L^{2}\left(0, T ; H^{s+1}\right)}^{2}+\|u\|_{L^{2}\left(0, T ; H^{s+1}\right)}^{2}\right) .
\end{gathered}
$$

Thus, we complete the proof.

\section{WG for primary integro-differential equation}

In this section, we firstly present several results regarding approximation properties of the $L^{2}$ projections $R_{h}$ and $Q_{h}$. Then, we provide the $H^{1}$ norm and $L^{2}$ norm for the linear integro-differential equation without the item $u_{t}$, respectively, to support the analysis of previous error estimates. 
Lemma 5.1 ([19]). Let $\mathcal{T}_{h}$ be a finite element partition of $\Omega$ satisfying the shape regularity [20] and $w \in H^{r+1}$ and $\rho \in H^{r}(\Omega)$ with $1 \leq r \leq k$. Then, for $0 \leq s \leq 1$ we have

$$
\begin{aligned}
& \sum_{T \in \mathcal{T}_{h}} h_{T}^{2 s}\left\|w-Q_{0} w\right\|_{T, s}^{2} \leq C h^{2(r+1)}\|w\|_{r+1}^{2}, \\
& \sum_{T \in \mathcal{T}_{h}} h_{T}^{2 s}\left\|\nabla w-Q_{0}(\nabla w)\right\|_{T, s}^{2} \leq C h^{2 r}\|w\|_{r+1}^{2} .
\end{aligned}
$$

Theorem 5.1. Let $u \in H^{s+1}(\Omega)$ be the exact solution of linear integro-differential equation without the item $u_{t}$. According to the definition of $\rho$, then

$$
\|\rho\| \| C h^{s}\left(\|u\|_{s+1}^{2}+\int_{0}^{t}\|u\|_{s+1}^{2} d \zeta\right)^{\frac{1}{2}}, \quad 0 \leq t \leq T .
$$

Proof. It follows from the definition of $E_{h}$ and integrating by parts with any $v \in V_{h}^{0}$ that

$$
\begin{aligned}
& a\left(A, E_{h} u, v\right)+\int_{0}^{t} a\left(B, E_{h} u, v\right) d \zeta \\
= & -\left(\nabla \cdot(A \nabla u), v_{0}\right)-\int_{0}^{t}\left(\nabla \cdot(B \nabla u), v_{0}\right) d \zeta \\
= & \left(A \nabla u, \nabla v_{0}\right)-\sum_{T \in \mathcal{T}_{h}}\left\langle A \nabla u \cdot \mathbf{n}, v_{0}-v_{b}\right\rangle_{\partial T}+\int_{0}^{t}\left(B \nabla u, \nabla v_{0}\right) d \zeta \\
& -\int_{0}^{t} \sum_{T \in \mathcal{T}_{h}}\left\langle B \nabla u \cdot \mathbf{n}, v_{0}-v_{b}\right\rangle_{\partial T} d \zeta .
\end{aligned}
$$

By the definition of $a(\cdot, \cdot, \cdot)$ and discrete weak gradient, commutativity and integrating by parts, we have

$$
\begin{aligned}
& a\left(A, Q_{h} u, v\right)+\int_{0}^{t} a\left(B, Q_{h} u, v\right) d \zeta \\
= & \left(A R_{h}(\nabla u), \nabla_{w} v\right)+\int_{0}^{t}\left(B R_{h}(\nabla u), \nabla_{w} v\right) d \zeta+s\left(Q_{h} u, v\right)+\int_{0}^{t} s\left(Q_{h} u, v\right) d \zeta \\
= & \left(A R_{h}(\nabla u), \nabla v_{0}\right)+\sum_{T \in \mathcal{T}_{h}}\left\langle A R_{h}(\nabla u) \cdot \mathbf{n}, v_{b}-v_{0}\right\rangle_{\partial T}+s\left(Q_{h} u, v\right)+\int_{0}^{t} s\left(Q_{h} u, v\right) d \zeta \\
& +\int_{0}^{t}\left(B R_{h}(\nabla u), \nabla v_{0}\right) d \zeta+\int_{0}^{t} \sum_{T \in \mathcal{T}_{h}}\left\langle B R_{h}(\nabla u) \cdot \mathbf{n}, v_{b}-v_{0}\right\rangle_{\partial T} d \zeta .
\end{aligned}
$$

From the above two equations, we obtain

$$
\begin{aligned}
& a(A, \rho, v)+\int_{0}^{t} a(B, \rho, v) d \zeta \\
= & \left(A\left(R_{h}(\nabla u)-\nabla u\right), \nabla v_{0}\right)+\int_{0}^{t}\left(B\left(R_{h}(\nabla u)-\nabla u\right), \nabla v_{0}\right) d \zeta \\
& \quad+l(A, u, v)+\int_{0}^{t} l(B, u, v) d \zeta+s\left(Q_{h} u, v\right)+\int_{0}^{t} s\left(Q_{h} u, v\right) d \zeta,
\end{aligned}
$$


where

$$
l(S, u, v)=\sum_{T \in \mathcal{T}_{h}}\left\langle S\left(R_{h}(\nabla u)-\nabla u\right) \cdot \mathbf{n}, v_{b}-v_{0}\right\rangle_{\partial T} .
$$

Taking $v=\rho$ in the error equation (5.2), we get

$$
\begin{aligned}
a(A, \rho, \rho)=- & \int_{0}^{t} a(B, \rho, \rho) d \zeta+\left(A\left(R_{h}(\nabla u)-\nabla u\right), \nabla \rho_{0}\right)+\int_{0}^{t} l(B, u, \rho) d \zeta \\
& +l(A, u, \rho)+s\left(Q_{h} u, \rho\right)+\int_{0}^{t}\left(B\left(R_{h}(\nabla u)-\nabla u\right), \nabla \rho_{0}\right) d \zeta \\
& +\int_{0}^{t} s\left(Q_{h} u, \rho\right) d \zeta .
\end{aligned}
$$

The properties of $A$ and $B$, the Cauchy-Schwarz, approximation properties and the trace inequality yield

$$
\begin{aligned}
&\|a(A, \rho, \rho)\| \geq \alpha_{1}|\|\rho\||^{2}, \\
&|l(A, u, \rho)|=\left|\sum_{T \in \mathcal{T}_{h}}\left\langle A\left(R_{h}(\nabla u)-\nabla u\right) \cdot \mathbf{n}, \rho_{0}-\rho_{b}\right\rangle_{\partial T}\right| \\
& \leq C\left|\|\rho \mid\| \cdot\left(\sum_{T \in \mathcal{T}_{h}}\left\|R_{h}(\nabla u)-u\right\|_{T}^{2}+h^{2}\left\|\nabla\left(R_{h}(\nabla u)-u\right)\right\|_{T}^{2}\right)^{1 / 2}\right. \\
& \leq C h^{2 s}\|u\|_{s+1}^{2}+\frac{\alpha_{1}}{6}|\|\rho\||^{2}, \\
&\left|s\left(Q_{h} u, \rho\right)\right|=\left|\sum_{T \in \mathcal{T}_{h}} h^{-1}\left\langle Q_{0} u-Q_{b} u, \rho_{0}-\rho_{b}\right\rangle_{\partial T}\right| \\
& \leq\left|\sum_{T \in \mathcal{T}_{h}} h^{-1}\left\langle Q_{0} u-u, \rho_{0}-\rho_{b}\right\rangle_{\partial T}\right| \\
& \leq C h^{2 s}\|u\|_{s+1}^{2}+\frac{\alpha_{1}}{6}\|\rho\|^{2},
\end{aligned}
$$

and

$$
\begin{aligned}
& \left\|\left(A\left(R_{h}(\nabla u)-\nabla u\right), \nabla \rho_{0}\right)\right\| \leq C\left\|R_{h}(\nabla u)-\nabla u\right\| \cdot\left\|\nabla \rho_{0}\right\| \\
& \quad \leq C h^{2 s}\|u\|_{s+1}^{2}+\frac{\alpha_{1}}{6}\|\rho\|^{2}, \\
& \left|\int_{0}^{t}\left(B\left(R_{h}(\nabla u)-\nabla u\right), \nabla \rho_{0}\right) d \zeta\right| \leq C h^{2 s} \int_{0}^{t}\|u\|_{s+1}^{2} d \zeta+C \int_{0}^{t}\|\| \rho \|\left.\right|^{2} d \zeta, \\
& \left|-\int_{0}^{t} a(B, \rho, \rho) d \zeta\right| \leq \beta_{1} \int_{0}^{t} \mid\|\rho\|^{2} d \zeta, \\
& \left|\int_{0}^{t} l(A, u, \rho) d \zeta\right| \leq C h^{2 s} \int_{0}^{t}\|u\|_{s+1}^{2} d \zeta+C \int_{0}^{t}\|\rho \mid\|^{2} d \zeta, \\
& \left|\int_{0}^{t} s\left(Q_{h} u, \rho\right) d \zeta\right| \leq C h^{2 s} \int_{0}^{t}\|u\|_{s+1}^{2} d \zeta+C \int_{0}^{t}\|\rho\|^{2} d \zeta .
\end{aligned}
$$


Combining these inequalities, we get

$$
\|\rho\|^{2} \leq C \int_{0}^{t}\|\rho\|\left\|^{2} d \zeta+C h^{2 s}\right\| u\left\|_{s+1}^{2}+C h^{2 s} \int_{0}^{t}\right\| u \|_{s+1}^{2} d \zeta .
$$

Using the Gronwall inequality, we obtain the error estimate in $H^{1}$ norm.

Next, we estimate $\|\rho\|$. Assume $\omega \in H^{1}(\Omega)$ is the exact solution of the elliptic problem with a Drichlet boundary condition

$$
\begin{array}{ll}
-\nabla \cdot(A \nabla \omega)=\rho_{0} & \text { in } \Omega, \\
\omega=0 & \text { on } \partial \Omega .
\end{array}
$$

We presume that the dual problem has the $H^{1}(\Omega)$-regularity property

$$
\|\omega\|_{2} \leq C\left\|\rho_{0}\right\| \text {. }
$$

Theorem 5.2. Let $u \in H^{s+1}(\Omega)$ be the exact solution of linear integro-differential equation without the term $u_{t}$. We have the following error estimate for $\rho_{0}$ :

$$
\left\|\rho_{0}\right\| \leq C h^{s+1}\left(\|u\|_{s+1}^{2}+\int_{0}^{t}\|u\|_{s+1}^{2} d \zeta\right)^{\frac{1}{2}}, \quad 0 \leq t \leq T .
$$

Proof. Testing the elliptic problem (5.3a) against $\rho_{0}$, we have

$$
\begin{aligned}
\left\|\rho_{0}\right\|^{2}= & \left(-\nabla \cdot(A \nabla \omega), \rho_{0}\right)=\left(A \nabla \omega, \nabla \rho_{0}\right)-\sum_{T \in \mathcal{T}_{h}}\left\langle A \nabla \omega \cdot n, \rho_{0}\right\rangle_{\partial T} \\
= & \left((A-\bar{A})\left(\nabla \omega-R_{h}(\nabla \omega)\right), \nabla \rho_{0}\right)+\left(A R_{h}(\nabla \omega), \nabla \rho_{0}\right) \\
& \quad-\sum_{T \in \mathcal{T}_{h}}\left\langle A \nabla \omega \cdot n, \rho_{0}-\rho_{b}\right\rangle_{\partial T} \\
= & \left((A-\bar{A})\left(\nabla \omega-R_{h}(\nabla \omega)\right), \nabla \rho_{0}\right)+a\left(A, Q_{h} \omega, \rho\right) \\
& \quad-s\left(Q_{h} \omega, \rho\right)-l(A, \omega, \rho),
\end{aligned}
$$

where using the fact $\left(\bar{A}\left(\nabla \omega-R_{h} \nabla \omega\right), \rho_{0}\right)=0$ and $\sum_{T \in \mathcal{T}_{h}}\left\langle A \nabla \omega \cdot n, \rho_{b}\right\rangle=0$. Considering Eq. (5.5) and taking $v=Q_{h} \omega$ in the error equation (5.2), we obtain

$$
\begin{aligned}
\left\|\rho_{0}\right\|^{2}= & -\int_{0}^{t}\left(B \nabla_{w} \rho, R_{h}(\nabla \omega)-\nabla \omega\right) d \zeta-\int_{0}^{t}\left(\nabla_{w} \rho, B \nabla \omega\right) d \zeta \\
& -\int_{0}^{t} s\left(\rho, Q_{h} \omega\right) d \zeta+\left((A-\bar{A})\left(\nabla \omega-R_{h}(\nabla \omega), \nabla \rho_{0}\right)\right. \\
& +l(A, \omega, \rho)-s\left(Q_{h} \omega, \rho\right)-\left((A-\bar{A})\left(\nabla u-R_{h}(\nabla u)\right), \nabla\left(Q_{0} \omega\right)\right) \\
& -\int_{0}^{t}\left((B-\bar{B})\left(\nabla u-R_{h}(\nabla u)\right), \nabla\left(Q_{0} \omega\right)\right) d \zeta+l\left(A, u, Q_{h} \omega\right) \\
& +\int_{0}^{t} l\left(B, u, Q_{h} \omega\right) d \zeta+s\left(Q_{h} u, Q_{h} \omega\right)+\int_{0}^{t} s\left(Q_{h} u, Q_{h} \omega\right) d \zeta .
\end{aligned}
$$


In order to get the estimates, we estimate the form term by term. Using the definition of the discrete weak gradient, the trace inequality and the property of projection yield

$$
\begin{aligned}
& \left|\int_{0}^{t}\left(B \nabla_{w} \rho, R_{h}(\nabla \omega)-\nabla \omega\right) d \zeta\right| \leq C \int_{0}^{t}\left\|\nabla_{w} \rho\right\|\left\|R_{h}(\nabla \omega)-\nabla \omega\right\| d \zeta \\
& \quad \leq C h \int_{0}^{t}\|\omega\|_{2}|\|\rho \mid\| d \zeta, \\
& \left\|\int_{0}^{t}\left(\nabla_{w} \rho, B \nabla \omega\right) d \zeta\right\|=\left\|\int_{0}^{t}\left(\rho_{0}, \nabla \cdot(B \nabla \omega)\right) d \zeta\right\| \leq \int_{0}^{t}\left\|\rho_{0}\right\|\|\omega\|_{2} d \zeta, \\
& \left|(A-\bar{A})\left(\nabla \omega-R_{h}(\nabla \omega)\right), \nabla \rho_{0}\right| \leq C h^{2}\|A\|_{1, \infty} \cdot\|\omega\|_{2}\|\rho\| \|,
\end{aligned}
$$

and

$$
\begin{aligned}
& \left|s\left(Q_{h} u, Q_{h} \omega\right)\right| \leq C\left(\sum_{T \in \mathcal{T}_{h}} h_{T}^{-1}\left\|Q_{0} u-u\right\|_{\partial T}^{2}\right)^{\frac{1}{2}}\left(\sum_{T \in \mathcal{T}_{h}} h_{T}^{-1}\left\|Q_{0} \omega-\omega+\omega-Q_{b} \omega\right\|_{\partial T}^{2}\right)^{\frac{1}{2}} \\
& \quad \leq C h^{s+1}\|u\|_{s+1}\|\omega\|_{2} \\
& \quad l l\left(A, u, Q_{h} \omega\right)|=| \sum_{T \in \mathcal{T}_{h}}\left\langle A\left(R_{h}(\nabla u)-\nabla u\right) \cdot \mathbf{n}, Q_{0} \omega-\omega+\omega-Q_{b} \omega\right\rangle_{\partial T} \mid \\
& \quad \leq C h^{s+1}\|u\|_{s+1}\|\omega\|_{2} .
\end{aligned}
$$

Following the estimate of $\|\mid \rho\| \|$, the definition of the discrete weak gradient, the trace inequality and the property of projection, we have

$$
\begin{aligned}
& \left|s\left(Q_{h} \omega, \rho\right)\right| \leq C h\|\omega\|_{2}\left|\left\|\rho \left|\| , \quad | l ( A , \omega , \rho ) | \leq C h \| \omega \left\|_{2}|\|\rho\||,\right.\right.\right.\right. \\
& \left|\int_{0}^{t}\left((B-\bar{B})\left(\nabla u-R_{h}(\nabla u)\right), \nabla\left(Q_{0} \omega\right)\right) d \zeta\right| \leq C h \int_{0}^{t}\|\omega\|_{2}\|\rho\| d \zeta, \\
& \left|\left((A-\bar{A})\left(\nabla u-R_{h}(\nabla u)\right), \nabla\left(Q_{0} \omega\right)\right)\right| \leq C h^{s+1}\|\omega\|_{2}\|u\|_{s+1},
\end{aligned}
$$

and

$$
\begin{aligned}
& \left|\int_{0}^{t} s\left(Q_{h} u, Q_{h} \omega\right) d \zeta\right| \leq C h^{s+1} \int_{0}^{t}\|u\|_{s+1}\|\omega\|_{2} d \zeta, \\
& \left|\int_{0}^{t} s\left(\rho, Q_{h} \omega\right) d \zeta\right| \leq C h \int_{0}^{t}\|\rho\|\|\omega\|_{2} d \zeta, \\
& \left|\int_{0}^{t} l\left(B, u, Q_{h} \omega\right) d \zeta\right| \leq C h^{s+1} \int_{0}^{t}\|u\|_{s+1}\|\omega\|_{2} d \zeta .
\end{aligned}
$$

From the estimate of $\|\mid \rho\|$, regularity property and the Cauchy-Schwarz inequality, we get

$$
\left\|\rho_{0}\right\|^{2} \leq \frac{1}{2}\left\|\rho_{0}\right\|^{2}+C \int_{0}^{t}\left\|\rho_{0}\right\|^{2} d \zeta+C h^{s+1}\left(\|u\|_{s+1}^{2}+\int_{0}^{t}\|u\|_{s+1}^{2} d \zeta\right)
$$


According to the Gronwall inequality, we obtain

$$
\left\|\rho_{0}\right\| \leq C h^{s+1}\left(\|u\|_{s+1}^{2}+\int_{0}^{t}\|u\|_{s+1}^{2} d \zeta\right)^{\frac{1}{2}} .
$$

The proof of this theorem is completed.

Similar to the proof of Theorem 5.1 and Theorem 5.2, we can obtain the estimate of $\rho_{t}$.

Theorem 5.3. If $u_{t} \in L^{\infty}\left(0, T ; H^{s+1}(\Omega)\right)$, there is a positive $C$ with $0 \leq t \leq T$ such that

$$
\begin{aligned}
& \left\|\mid \rho_{t}\right\| \leq \leq C h^{s}\left(\|u\|_{s+1}^{2}+\left\|u_{t}\right\|_{s+1}^{2}+\int_{0}^{t}\left(\|u\|_{s+1}^{2}+\left\|u_{t}\right\|_{s+1}^{2}\right) d \zeta\right)^{\frac{1}{2}}, \\
& \left\|\left(\rho_{0}\right)_{t}\right\| \leq C h^{s+1}\left(\|u\|_{s+1}^{2}+\left\|u_{t}\right\|_{s+1}^{2}+\int_{0}^{t}\left(\|u\|_{s+1}^{2}+\left\|u_{t}\right\|_{s+1}^{2}\right) d \zeta\right)^{\frac{1}{2}} .
\end{aligned}
$$

\section{Numerical example}

We present several numerical examples to verity the order of convergence with weak Galerkin finite element method by adding the stabilizer for the linear parabolic integrodifferential equation (1.1a)-(1.1c).

Example 6.1. We solve (1.1a) over the square domain $\Omega=(0,1) \times(0,1)$ where

$$
\begin{aligned}
& A=\left(\begin{array}{cc}
3+x-y & 1 / 2 \\
1 / 2 & 4-x+y
\end{array}\right), \quad B=\left(\begin{array}{cc}
x+y & -1 / 2 \\
-1 / 2 & x+y
\end{array}\right), \\
& \Omega=(0,1) \times(0,1),
\end{aligned}
$$

and the exact solution is chosen as

$$
u(x, y, t)=e^{-t} x(1-x) y(1-y) .
$$

We use $P_{k}$ weak Galerkin finite elements on rectangular grids where the first level grid is the domain itself and each grid is refined in to the half-sized grid to form the next level grid. We choose $\tau=10^{-4}$ and compute the solution up to $T=1$. The errors and the orders of convergence are listed in Table 1.

Example 6.2. We solve (1.1a) where

$$
\begin{aligned}
& A=\left(\begin{array}{ll}
1 & 0 \\
0 & 1
\end{array}\right), \quad B=\beta_{1}\left(\begin{array}{cc}
0 & -1 / 2 \\
-1 / 2 & 0
\end{array}\right), \quad \beta_{1}=1 \quad \text { or } 10, \\
& f=1, \quad g=0, \quad \psi=0, \quad \Omega \times(0, T)=(0,1) \times(0,1) \times(0,1) .
\end{aligned}
$$

We do not know the exact solution. The numerical solutions are plotted in Fig. 1, where we can see the effect of memory integral. 
Table 1: Example 6.1. The errors and the orders of convergence for solving (6.1).

\begin{tabular}{||c|cc|cc|c|c||}
\hline $\mathcal{T}_{k}$ & $\left\|Q_{h} u-u_{h}\right\|_{0}$ & $h^{n}$ & $\left\|Q_{h} u-u_{h}\right\|_{1, h}$ & $h^{h}$ & $\left\|\mid Q_{h} u-u_{h}\right\| \|$ & $h^{n}$ \\
\hline \multicolumn{7}{|c||}{$P_{1}$ weak Galerkin finite element method } \\
\hline 4 & $0.1528 \mathrm{E}-02$ & 1.90 & $0.7005 \mathrm{E}-02$ & 1.08 & $0.4221 \mathrm{E}-02$ & 1.34 \\
5 & $0.3889 \mathrm{E}-03$ & 1.97 & $0.3601 \mathrm{E}-02$ & 0.96 & $0.1176 \mathrm{E}-02$ & 1.84 \\
6 & $0.9743 \mathrm{E}-04$ & 2.00 & $0.1876 \mathrm{E}-02$ & 0.94 & $0.3031 \mathrm{E}-03$ & 1.96 \\
\hline \multicolumn{7}{|c||}{$P_{2}$ weak Galerkin finite element method } \\
\hline 4 & $0.6438 \mathrm{E}-04$ & 3.07 & $0.1997 \mathrm{E}-02$ & 2.04 & $0.1397 \mathrm{E}-02$ & 2.27 \\
5 & $0.7492 \mathrm{E}-05$ & 3.10 & $0.4782 \mathrm{E}-03$ & 2.06 & $0.2871 \mathrm{E}-03$ & 2.28 \\
6 & $0.9107 \mathrm{E}-06$ & 3.04 & $0.1161 \mathrm{E}-03$ & 2.04 & $0.5943 \mathrm{E}-04$ & 2.27 \\
\hline \multicolumn{7}{|c||}{$P_{3}$ weak Galerkin finite element method } \\
\hline 2 & $0.1048 \mathrm{E}-02$ & 3.70 & $0.1140 \mathrm{E}-01$ & 2.55 & $0.1315 \mathrm{E}-01$ & 2.93 \\
3 & $0.6760 \mathrm{E}-04$ & 3.95 & $0.1529 \mathrm{E}-02$ & 2.90 & $0.1657 \mathrm{E}-02$ & 2.99 \\
4 & $0.4138 \mathrm{E}-05$ & 4.03 & $0.1891 \mathrm{E}-03$ & 3.02 & $0.1967 \mathrm{E}-03$ & 3.07 \\
\hline \multicolumn{7}{|c|}{$P_{4}$ weak Galerkin finite element method } \\
\hline 2 & $0.9909 \mathrm{E}-04$ & 5.06 & $0.1826 \mathrm{E}-02$ & 4.07 & $0.2976 \mathrm{E}-03$ & 2.94 \\
3 & $0.3014 \mathrm{E}-05$ & 5.04 & $0.1079 \mathrm{E}-03$ & 4.08 & $0.2582 \mathrm{E}-04$ & 3.53 \\
4 & $0.1551 \mathrm{E}-06$ & 4.28 & $0.6457 \mathrm{E}-05$ & 4.06 & $0.1910 \mathrm{E}-05$ & 3.76 \\
\hline
\end{tabular}
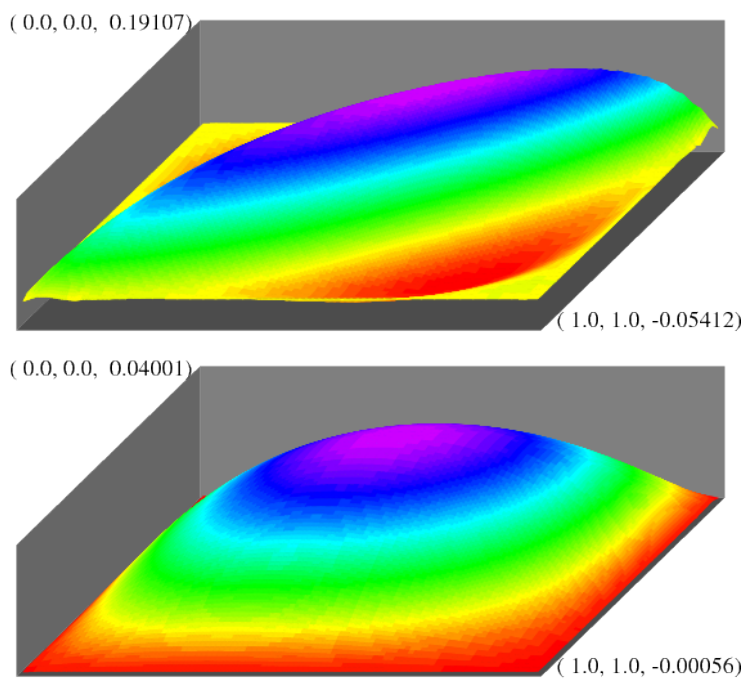

Figure 1: Example 6.2. The weak Galerkin solution for $\beta=1$ (bottom) and for $\beta=10$ (top).

\section{Conclusions}

In this paper, we developed another weak Galerkin finite element method with a stabilizer, which provides more options to choose elements of partition, for solving linear parabolic and primary integro-differential problems. The semi-discrete and fully-discrete weak Galerkin finite element schemes were constructed. The semi-discrete WG scheme 
was proved to be stable with respect to the right hand side. The fully-discrete WG scheme was discretized by the backward Euler method. The optimal orders of convergence were obtained in $L^{2}$ and $H^{1}$ norms. Numerical examples confirmed theoretical analysis.

\section{Acknowledgements}

The research of Qilong Zhai was supported in part by China Natural National Science Foundation (No. 11901015) and China Postdoctoral Science Foundation (Nos. 2018M640013 and 2019T120008). The research of Ran Zhang was supported in part by China Natural National Science Foundation (Nos. 91630201, U1530116, 11726102, 11771179, 93K172018Z01, 11701210, JJKH20180113KJ and 20190103029JH) and by the Program for Cheung Kong Scholars of Ministry of Education of China, Key Laboratory of Symbolic Computation and Knowledge Engineering of Ministry of Education.

\section{References}

[1] BASHIR AHMAD AND S SIVASUndARAm, Dynamics and stability of impulsive hybrid setvalued integro-differential equations with delay, Nonlinear Anal. Theory, Methods Appl., 65(11) (2006), pp. 2082-2093.

[2] Hongbo CHEn And Tianliang Hou, A priori and a posteriori error estimates of $H^{1}$-Galerkin mixed finite element methods for optimal control problems governed by pseudo-hyperbolic integrodifferential equations, Appl. Math. Comput., 328 (2018), pp. 100-112.

[3] GAMAL N. EDIT, Optimal control computation for integro-differential aerodynamic equations, Math. Methods Appl. Sci., 21(7) (1998), pp. 653-664.

[4] DeEpjyoti Goswami, Amiya K. PANi AND SAngita YADAV, Optimal error estimates of two mixed finite element methods for parabolic integro-differential equations with nonsmooth initial data, J. Sci. Comput., 56(1) (2013), pp. 131-164.

[5] STIG LARSSON, VIDAR THOMÉE AND LARS B. WAHLBIN, Numerical solution of parabolic integro-differential equations by the discontinuous Galerkin method, Math. Comput. Am. Math. Soc., 67(221) (1998), pp. 45-71.

[6] JingZHi Li, XiAOSHEN WANG AND KAI ZHANG, Multi-level Monte Carlo weak Galerkin method for elliptic equations with stochastic jump coefficients, Appl. Math. Comput., 275 (2016), pp. 181-194.

[7] ZHiHUi LiU AND ZHONGHUA QIAO, WongZakai approximations of stochastic Allen-Cahn equation, Int. J. Numer. Anal. Model., 16(5) (2019), pp. 681-694.

[8] F. G. MAKSUdOV AND T. M. RASUlOV, Solution of problems for a nonlinear system of integrodifferential equations that characterizes the process of displacement of petroleum from strata by mineral, Azerbaidzhan. Gos. Univ. Baku, 12(7) (1981), pp. 66-70.

[9] SEAN MCKEE, Volterra integral and integro-differential equations arising from problems in engineering and science, Bull. Inst. Math. Appl., 24(9-10) (1988), pp. 135-138.

[10] ABDELAZIZ MENNOUNI, Airfoil polynomials for solving integro-differential equations with logarithmic kernel, Appl. Math. Comput., 218(24) (2012), pp. 11947-11951. 
[11] K. Mustapha, H. Brunner, H. Mustapha and D. Schötzau, An hp-version discontinuous Galerkin method for integro-differential equations of parabolic type, SIAM J. Numer. Anal., 48(4) (2011), pp. 1369-1396.

[12] K. S. SCHMID, S. GEIGER AND K. S. SORBIE, Higher order FE-FV method on unstructured grids for transport and two-phase flow with variable viscosity in heterogeneous porous media, J. Comput. Phys., 241 (2013), pp. 416-444.

[13] ZHONGHUA QIAO, A new multi-component diffuse interface model with Peng-Robinson equation of state and its scalar auxiliary variable (SAV) approach, Commun. Comput. Phys., 26(5) (2019), pp. 1597-1616.

[14] FATEMEH SHAKeRI AND MeHDi DeHGHAN, A high order finite volume element method for solving elliptic partial integro-differential equations, Appl. Numer. Math., 65 (2013), pp. 105-118.

[15] A. S. C. SINHA, Integro-differential equations arising in population dynamics and control systems, Int. J. Control, 27(4) (1978), pp. 571-577.

[16] Raytcho D. Sinha, Rajen K. EWing And Richard E. LAZAROV, Some new error estimates of a semidiscrete finite volume element method for a parabolic integro-differential equation with nonsmooth initial data, SIAM J. Numer. Anal., 43(6) (2006), pp. 2320-2343.

[17] TIAn TIAN, QILONG ZHAi AND RAN ZHANG, A new modified weak Galerkin finite element scheme for solving the stationary Stokes equations, J. Comput. Appl. Math., 329 (2018), pp. 268279.

[18] JUNPING WANG, RUISHU WANG, QILONG Zhai AND RAN ZHANG, A systematic study on weak galerkin finite element methods for second order elliptic problems, J. Sci. Comput., 74(3) (2018), pp. 1369-1396.

[19] JUNPING WANG AND XIU YE, A weak Galerkin finite element method for second-order elliptic problems, J. Comput. Appl. Math., 241(1) (2013), pp. 103-115.

[20] JUNPING WANG AND XIU YE, A weak Galerkin finite element method for the stokes equations, Adv. Comput. Math., 42(1) (2016), pp. 155-174.

[21] XIUli WANG, A class of weak Galerkin finite element methods for the incompressible fluid model, Adv. Appl. Math. Mech., 11(2) (2019), pp. 360-380.

[22] XiUli WANG, QILONG Zhai, RUishU WANG AND RabeEa Jari, An absolutely stable weak Galerkin finite element method for the Darcy-Stokes problem, Appl. Math. Comput., 331 (2018), pp. 20-32.

[23] Xiuli Wang, Qilong Zhai And RAn Zhang, The weak Galerkin method for solving the incompressible Brinkman flow, J. Comput. Appl. Math., 307 (2016), pp. 13-24.

[24] XINGFA YANG, Jacobi spectral collocation method based on Lagrange interpolation polynomials for solving nonlinear fractional integro-differential equations, Adv. Appl. Math. Mech., 10(6) (2018), pp. 1440-1458.

[25] Elizabeth G. YANiK AND Graeme Fairweather, Finite element methods for parabolic and hyperbolic partial integro-differential equations, Nonlinear Anal. Theory Methods Appl., 12(8) (1988), pp. 785-809.

[26] Qilong Zhai, Hehu Xie, RAn Zhang And Zhimin Zhang, The weak Galerkin method for elliptic eigenvalue problems, Commun. Comput. Phys., 26(1) (2019), pp. 160-191.

[27] QILONG ZHAI, RAN ZHANG AND LIN MU, A new weak Galerkin finite element scheme for the Brinkman model, Commun. Comput. Phys., 19(05) (2016), pp. 1409-1434.

[28] QILONG ZHAI, RAN ZHANG AND XIAOSHEN WANG, A hybridized weak Galerkin finite element scheme for the Stokes equations, Sci. China Math., 58(11) (2015), pp. 2455-2472.

[29] HongQin Zhang, YongKui Zou, YingXIANG XU, QILONG ZHAi AND HuA Yue, Weak Galerkin finite element method for second order parabolic equations, Int. J. Numer. Anal. Model., 
13(4) (2016), pp. 525-544.

[30] RAN ZHANG AND QILONG ZHAI, A weak Galerkin finite element scheme for the Biharmonic equations by using polynomials of reduced order, J. Sci. Comput., 64(2) (2015), pp. 559-585.

[31] WeISHAN ZHENG, Convergence analysis of Legendre-Collocation spectral methods for second order Volterra integro-differential equation with delay, Adv. Appl. Math. Mech., 11(2) (2019), pp. 486500.

[32] Ailing Zhu, TINGTING XU AND QIANG XU, Weak Galerkin finite element methods for linear parabolic integro-differential equations, Numer. Methods Partial Differential Equations, 32(5) (2016), pp. 1357-1377.

[33] HongZe Zhu, Yongkui Zou, Shimin Chai And Chenguang Zhou, Numerical approximation to a stochastic parabolic PDE with weak Galerkin method, Numer. Math. Theory Methods Appl., 11(3) (2018), pp. 604-617. 